\title{
Antinucleon-nucleon interaction in chiral effective field theory
}

\author{
Johann Haidenbauer ${ }^{1, \star}$ \\ ${ }^{1}$ Institute for Advanced Simulation, Institut für Kernphysik, and Jülich Center for Hadron Physics, \\ Forschungszentrum Jülich GmbH, D-52425 Jülich, Germany
}

\begin{abstract}
A study of the antinucleon-nucleon interaction within chiral effective field theory is presented. This novel approach suggested by Weinberg for investigating the nucleon-nucleon interaction can be adapted straightforwardly to the antinucleon-nucleon system. The antinucleon-nucleon potential is derived up to next-to-next-to-next-toleading order in the chiral expansion. The low-energy constants associated with the arising contact interactions are fixed by a fit to phase shifts and inelasticities provided by a recently published phase-shift analysis of antiproton-proton scattering data. The achieved description of the antinucleon-nucleon amplitudes is excellent and of a quality comparable to the one found in case of the nucleon-nucleon interaction at the same order.
\end{abstract}

\section{Introduction}

Over the last decade there has been a renewed interest in antinucleon-nucleon $(\bar{N} N)$ scattering phenomena. It was prompted not least by measurements of the $\bar{p} p$ invariant mass in the decays of heavy mesons such as $J / \psi, \psi^{\prime}$, and $B$, and of the reaction cross section for $e^{+} e^{-} \rightarrow \bar{p} p$. In several of those reactions a sizeable near-threshold enhancement in the mass spectrum was found [1-4]. Those enhancements nourished speculations about $\bar{p} p$ bound states, new resonances, or even more exotic objects in some parts of the physics community. Others noted that such data could provide a unique opportunity to test the $\bar{p} p$ interaction at very low energies [5-11]. Another boost comes, of course, from activities related to the $\bar{N} N$ interaction planned for the Facility for Antiproton and Ion Research (FAIR) in Darmstadt whose construction is finally on its way. Specifically, the PANDA project aims at a study of the interactions between antiprotons and fixed target protons and nuclei in the momentum range of 1.5-15 GeV/c using the high energy storage ring HESR [12]. In this context also the proposal by the PAX Collaboration [13] should be mentioned, though it is not in the core program of FAIR. It intends to exploit the method of spin-filtering in storage rings to achieve a polarized antiproton beam which would then allow one to measure $\bar{N} N$ double-spin observables.

The renewed interest in $\bar{N} N$ phenomena led also to the construction of new $\bar{N} N$ potentials, phenomenological ones $[14,15]$ but also of interactions [16] that are based on modern and systematic approaches like chiral effective field theory (EFT) [17]. Indeed, one of the consequences of a growing interest in $\bar{N} N$ physics was perhaps that an update of the Nijmegen partial-wave analysis (PWA) of antiproton-proton scattering data has been presented [18]. Since for the new PWA the resulting phase shifts and inelasticities are explicitly given, a straightforward application of chiral EFT to studies of the $\bar{N} N$ force became feasible $[19,20]$.

^e-mail: j.haidenbauer@fz-juelich.de 
Chiral EFT has been suggested by Weinberg in the early 1990s [21] as a systematic approach for investigating nucleon-nucleon $(N N)$ scattering. It facilitates a rather accurate and consistent description of the $N N$ interaction and nuclear few-body systems [22, 23], see also the overviews in Refs. [17, 24]. Its most salient features are that it exploits the symmetries and symmetry-breaking pattern of QCD and that there is an underlying power counting which allows one to improve calculations systematically by going to higher orders in a perturbative expansion. The emerging chiral potential for the $N N$ force consists of pion exchanges and a series of contact interactions with an increasing number of derivatives. The latter represent the short-range part of the $N N$ force and are parameterized by low-energy constants (LECs), that need to be fixed by a fit to data. The reaction amplitude is obtained from solving a regularized Lippmann-Schwinger equation for the derived interaction potential.

The $\bar{N} N$ interaction is closely connected to that in the $N N$ system via $G$-parity. Specifically, the $G$-parity transformation (a combination of charge conjugation and a rotation in the isospin space) relates that part of the $\bar{N} N$ potential which is due to pion exchanges to the one in the $N N$ case in an unambiguous way. Thus, like in the $N N$ case, the long-range part of the $\bar{N} N$ potential is completely fixed by the underlying chiral symmetry of pion-nucleon dynamics.

In this contribution I review a $\bar{N} N$ potential derived in a chiral EFT approach up to next-to-nextto-next-to leading order $\left(\mathrm{N}^{3} \mathrm{LO}\right)$. Its evaluation has been done in complete analogy to that of the $N N$ interaction published in Ref. [23] and is likewise based on the Weinberg power counting. In Ref. [19] our group had already studied the $\bar{N} N$ force within chiral EFT up to next-to-next-to leading order $\left(\mathrm{N}^{2} \mathrm{LO}\right)$. It had been found that the approach works very well. Indeed, the overall quality of the description of the $\bar{N} N$ amplitudes achieved in Ref. [19] is comparable to the one found in case of the $N N$ interaction at the same order. By going to a higher order one expects to be able to describe the $\bar{N} N$ interaction over a larger energy range. Furthermore, in Ref. [23] a simple approach for estimating the uncertainty due to the truncation of the chiral expansion is proposed. This approach can be adopted for performing an analogous analysis for our $\bar{N} N$ results.

\section{The $\bar{N} N$ interaction in chiral EFT}

The derivation of the chiral $\bar{N} N$ potential using the Weinberg power counting is outlined in detail in Ref. [20], see also Ref. [23] for the corresponding application to the $N N$ system. Therefore, I will be very brief here. As already mentioned above, the chiral potential contains pion exchanges and a series of contact interactions with an increasing number of derivatives. Up to $\mathrm{N}^{3} \mathrm{LO}$ there are contributions from one-, two- and three-pion exchanges. Those are identical to the ones that appear in the $N N$ potential [23]. However, there is a sign change in case of an odd number of exchanged pions due to its negative $G$ parity, i.e. $V^{\bar{N} N}=-V_{\pi}+V_{2 \pi}-V_{3 \pi}$, with $V_{\pi}$ etc. being the corresponding contributions to the $N N$ force. On the other hand, the contact interaction, $V_{c o n t}^{\bar{N} N}$, cannot be taken over simply from the $N N$ case. As said above, the contact terms represent effectively the short-range part of the interaction and, therefore, the $G$ parity of the indivudal contributions remains unresolved. Thus, the strength parameters associated with the arising contact terms, the LECs, need to be determined in a fit to $\bar{N} N$ data. We fix them by fitting to the phase shifts and inelasticity parameters of the PWA of Zhou and Timmermans [18]. How this is done is described in detail in Ref. [20]. Note that there are more independent LECs in the $\bar{N} N$ case than in $N N$ because in the former system there is no restriction from the Pauli principle. In addition, in our approach $\bar{N} N$ annihilation is likewise parameterized by contact terms, see Refs. [19, 20] for explicit expressions. 
Once the potential is established, the reaction amplitude is obtained from the solution of a relativistic Lippmann-Schwinger (LS) equation. It reads in partial-wave projected form

$$
\begin{aligned}
T_{L^{\prime \prime} L^{\prime}}\left(p^{\prime \prime}, p^{\prime} ; E_{k}\right) & =V_{L^{\prime \prime} L^{\prime}}\left(p^{\prime \prime}, p^{\prime}\right) \\
& +\sum_{L} \int_{0}^{\infty} \frac{d p p^{2}}{(2 \pi)^{3}} V_{L^{\prime \prime} L}\left(p^{\prime \prime}, p\right) \frac{1}{2 E_{k}-2 E_{p}+i 0^{+}} T_{L L^{\prime}}\left(p, p^{\prime} ; E_{k}\right) .
\end{aligned}
$$

Here $p^{\prime \prime}$ and $p^{\prime}$ are the (moduli of the) center-of-mass $\bar{N} N$ momenta in the final and initial states, respectively, and $E_{k}=\sqrt{M_{p}^{2}+k^{2}}$, where $k$ is the on-shell momentum and We adopt a relativistic scattering equation so that our amplitudes fulfill the relativistic unitarity condition at any order, as done also in the $N N$ sector $[22,24]$. On the other hand, relativistic corrections to the potential are calculated order by order. They appear first at $\mathrm{N}^{3} \mathrm{LO}$ in the Weinberg scheme, see Appendix A in Ref. [20]. Note that the $\bar{N} N$ system can be in uncoupled spin-singlet or triplet waves (where $L^{\prime \prime}=L^{\prime}=L=J$ ) or in coupled partial waves (where $L^{\prime \prime}, L^{\prime}, L=J-1, J+1$ ).

Since the integral in the LS equation (1) is divergent for the chiral potentials [22, 24] a regularization needs to be introduced. We utilize the regularization scheme of Ref. [23], where a local regulator is used for the pion-exchange contributions and a nonlocal regulator for the contact terms:

$$
\begin{aligned}
& V_{n \pi}(q) \rightarrow V_{n \pi}(r) \times f_{R}(r) \rightarrow V_{n \pi}^{r e g}(q) ; \quad\left(\vec{q}=\vec{p}^{\prime}-\vec{p}\right) \\
& V_{\text {cont }}=V\left(p^{\prime}, p\right) \rightarrow V\left(p^{\prime}, p\right) \times f_{\Lambda}\left(p^{\prime}, p\right)=V_{\text {cont }}^{r e g} .
\end{aligned}
$$

The explict form of the regulation functions is $f_{R}(r)=\left[1-\exp \left(-r^{2} / R^{2}\right)\right]^{6}$ and $f_{\Lambda}\left(p, p^{\prime}\right)=\exp \left(-\left(p^{\prime 2}+\right.\right.$ $\left.p^{2}\right) / \Lambda^{2}$ ). The cutoff radius $R$ is varied in the range $R=0.7-1.2 \mathrm{fm}$ [23] where $\Lambda=2 / R$ is used for relating the momentum-space cutoff parameter with the cutoff radius.

Since the fit is done to phase shifts and inelasticity parameters of Ref. [18], which are given for definite isospins, at this stage the LS equation is solved in the isospin basis. For the calculation of observables we solve the LS equation in the particle basis, in order to incorporate the correct physical thresholds of the $\bar{p} p$ and $\bar{n} n$ channels. Note that the $\bar{n} n$ channel opens at the antiproton laboratory momentum of $p_{\text {lab }}=98.7 \mathrm{MeV} / \mathrm{c}\left(T_{\text {lab }}=5.18 \mathrm{MeV}\right)$. Furthermore, the Coulomb interaction in the $\bar{p} p$ channel is taken into account appropriately via the Vincent-Phatak method.

\section{Results}

A complete overview of our investigation of $\bar{N} N$ scattering up to $\mathrm{N}^{3} \mathrm{LO}$ in chiral EFT can be found in Ref. [20]. Here we focus on specific aspects and present only some exemplary results. One of those aspects is the influence of the regularization scheme. While ideally the results should be completely independent of the employed regularization, this cannot be achieved within the scheme [23] adopted in our study [20]. Nonetheless, one can monitor the effects of keeping the cutoff finite, and the artifacts introduced by that, by analyzing the residual cutoff dependence of the observables [23]. Pertinent results are presented in Fig. 1, where phase shifts are displayed for partial waves up to an orbital angular momentum of $L=2$. We use the spectral notation ${ }^{(2 S+1)} L_{J}$ and indicate the isospin $I$ separately. Note that instead of the inelasticity parameters the imaginary part of the phases and mixing angles are shown and subscripts $R$ and $I$ are used for $\delta$ in order to distinguish between the real and imaginary parts. The employed cutoffs are $R=0.7,0.8,0.9,1.0,1.1$, and $1.2 \mathrm{fm}$ and the results are based on the chiral potential up to $\mathrm{N}^{3} \mathrm{LO}$.

One can see that for most partial waves the cutoff dependence is fairly weak for $T_{\text {lab }} \lesssim 300 \mathrm{MeV}$ $\left(p_{\text {lab }} \lesssim 800 \mathrm{MeV} / \mathrm{c}\right)$. Indeed, the small residual cutoff dependence that we observe here is comparable 

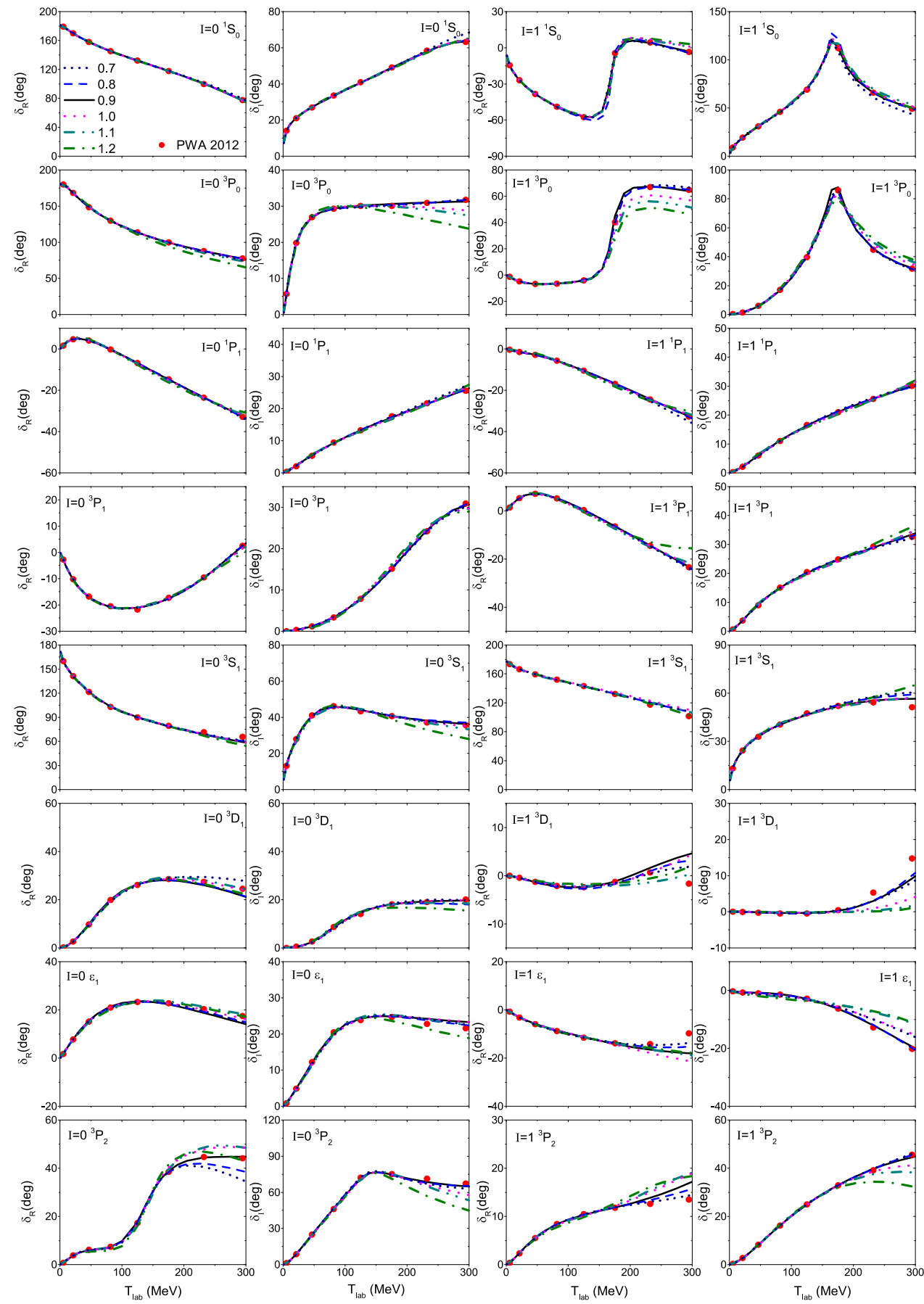

Figure 1. Real and imaginary parts of various $\bar{N} N$ phase shifts at $\mathrm{N}^{3} \mathrm{LO}$ for cutoffs $R=0.7-1.2 \mathrm{fm}$. The filled circles represent the solution of the $\bar{p} p$ PWA [18]. 
to the likewise small variation reported in Ref. [23] for the $N N$ interaction. Only in a few cases there is a more pronounced cutoff dependence of the results for laboratory energies above $150-200 \mathrm{MeV}$. This has to do with the fact that the PWA [18] suggests a resonance-like behavior of some phases in this region. This concerns most prominently the ${ }^{1} S_{0}$ partial wave with isospin $I=1$ and the ${ }^{3} P_{0}$ partial wave with $I=1$. In addition, also a few other partial waves show a conspicuous behavior at higher energies in the sense that the energy dependence changes noticeably. Typical examples are the inelasticity parameters for the $I=0{ }^{3} P_{0}$ and ${ }^{3} P_{2}$ partial wave, where the corresponding $\delta_{\mathrm{I}}$ increase rapidly from the threshold, but then level out at higher energies.

Results for partial waves up to a total angular momentum of $J=4$ can be found in Ref. [20].
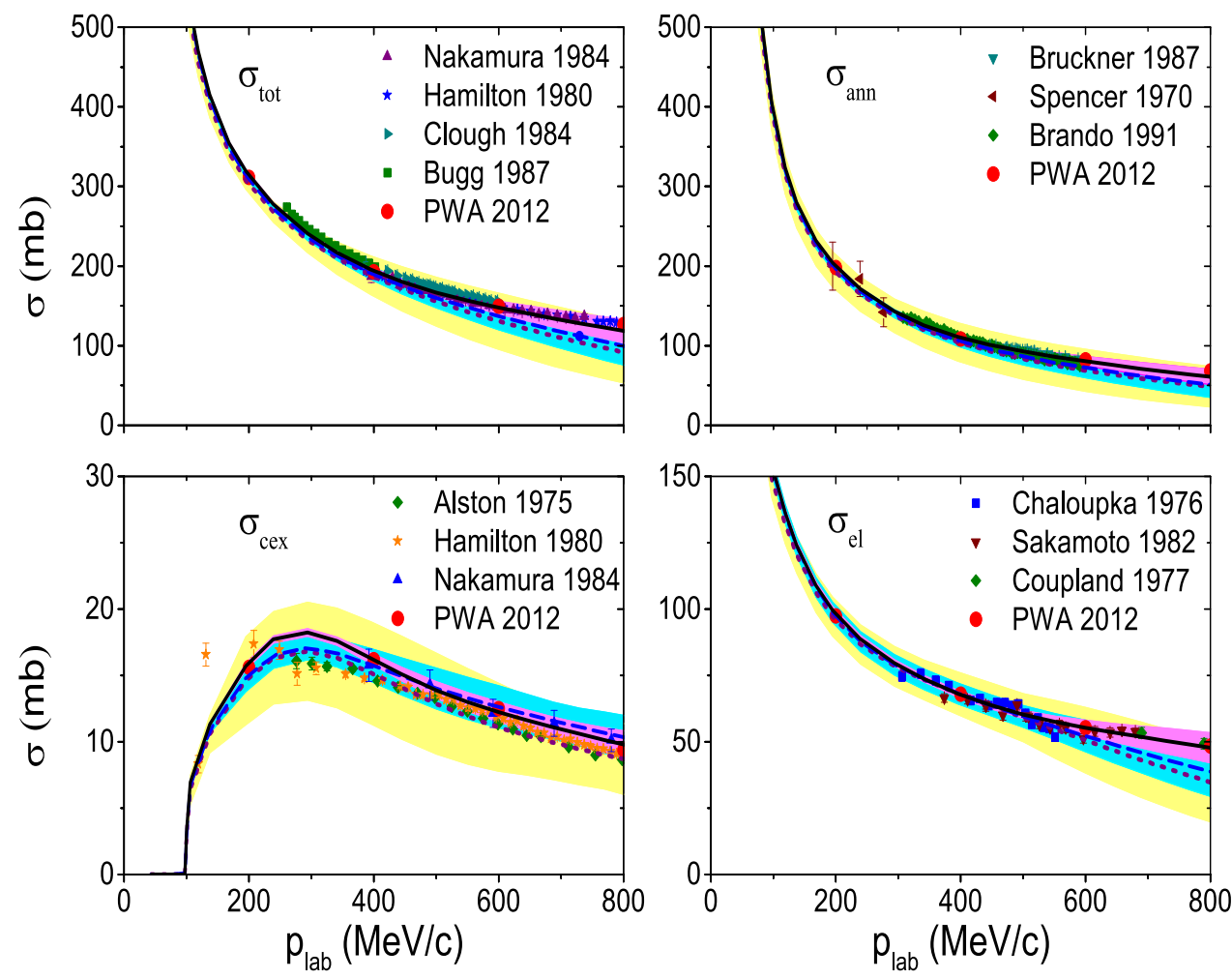

Figure 2. Total $\left(\sigma_{t o t}\right)$ and integrated elastic $\left(\sigma_{e l}\right)$, charge-exchange $\left(\sigma_{c e x}\right)$, and annihilation $\left(\sigma_{a n n}\right)$ cross sections for $\bar{p} p$ scattering. Results at $\mathrm{N}^{3} \mathrm{LO}$ (black solid line), $\mathrm{N}^{2} \mathrm{LO}$ (blue dashed line), and NLO (red dotted line) are shown. The estimated uncertainty is indicated by bands: $\mathrm{N}^{3} \mathrm{LO}$ (magenta), $\mathrm{N}^{2} \mathrm{LO}$ (cyan), and NLO (yellow). The filled circles represent the solution of the $\bar{p} p$ PWA [18].

Results for the integrated cross sections are presented in Fig. 2 for the chiral interaction with cutoff $R=0.9 \mathrm{fm}$ up to NLO (red dotted line), $\mathrm{N}^{2} \mathrm{LO}$ (blue dashed line), and $\mathrm{N}^{3} \mathrm{LO}$ (solid black line). Obviously, at $\mathrm{N}^{3} \mathrm{LO}$ our results agree rather well with the ones of the PWA (filled circles), even up to $p_{\text {lab }}=800 \mathrm{MeV} / \mathrm{c}$. Indeed, also the charge-exchange cross section is nicely reproduced, though it is much smaller than the other ones. The amplitude for this process is given by the difference of the isospin $I=0$ and $I=1$ amplitudes and its description requires a delicate balance between the interactions in the corresponding isospin channels. 

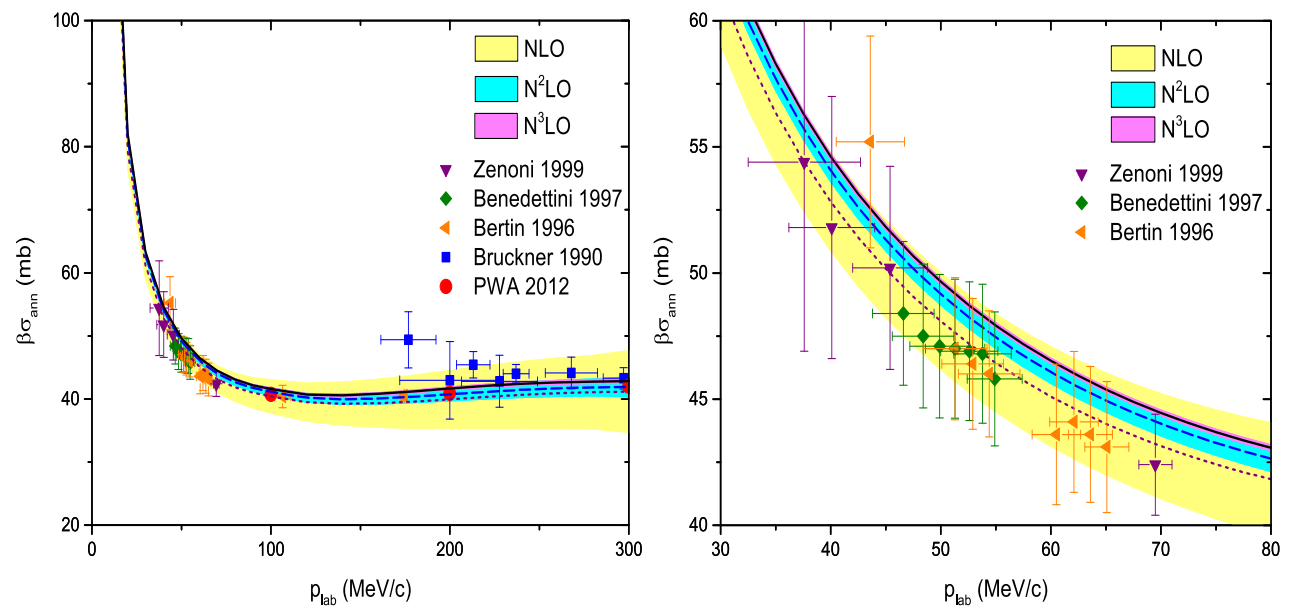

Figure 3. $\bar{p} p$ annihilation cross section multiplied by the velocity $\beta$ of the incoming $\bar{p}$. Same description of curves as in Fig. 2. The results of the PWA [18] are indicated by circles.

The bands represent the estimated uncertainty of our results. Here we follow the suggestion of Epelbaum et al. [23] and use as measure the expected size of the higher-order corrections together with the actual size of the higher-order corrections. Then the uncertainty of an observable $X(p)$ is given by

$$
\begin{aligned}
\Delta X^{L O} & =Q^{2}\left|X^{L O}\right| \\
\Delta X^{N L O} & =\max \left(Q^{3}\left|X^{L O}\right|, Q^{1}\left|\delta X^{N L O}\right|\right) ; \quad \delta X^{N L O}=X^{N L O}-X^{L O} \\
\Delta X^{N^{2} L O} & =\max \left(Q^{4}\left|X^{L O}\right|, Q^{2}\left|\delta X^{N L O}\right|, Q^{1}\left|\delta X^{N^{2} L O}\right|\right) ; \quad \delta X^{N^{2} L O}=X^{N^{2} L O}-X^{N L O} \\
\Delta X^{N^{3} L O} & =\max \left(Q^{5}\left|X^{L O}\right|, Q^{3}\left|\delta X^{N L O}\right|, Q^{2}\left|\delta X^{N^{2} L O}\right|, Q^{1}\left|\delta X^{N^{3} L O}\right|\right) ; \quad \delta X^{N^{3} L O}=X^{N^{3} L O}-X^{N^{2} L O},
\end{aligned}
$$

where $X^{L O}$, etc., are the results for that observable at the corresponding order. The expansion parameter $Q$ is defined by $Q=\max \left(\frac{k}{\Lambda_{b}}, \frac{m_{\pi}}{\Lambda_{b}}\right)$ with $k$ being the $\bar{N} N$ on-shell momentum. The quantity $\Lambda_{b}$ is the breakdown scale, i.e. the momentum where the chiral EFT expansion is expected to break down. For it we take over the value $\Lambda_{b}=600 \mathrm{MeV}$ from Ref. [23], deduced from the analysis of corresponding $N N$ results. Considering the bands for the interactions with increasing order - yellow (NLO), blue $\left(\mathrm{N}^{2} \mathrm{LO}\right)$, magenta $\left(\mathrm{N}^{3} \mathrm{LO}\right)$ - one can see that there is a clear convergence of our results for all cross sections when going to higher orders.

Results for differential observables, specifically for differential cross sections, analyzing powers, and depolarization parameters, can be found in Ref. [20], where they are compared directly with experimental information.

\section{Predictions}

As can be seen in Table III of Ref. [18] no data below $100 \mathrm{MeV} / \mathrm{c}$ (corresponding to $T_{\text {lab }}=5.32 \mathrm{MeV}$ ) have been included in the partial-wave analysis. Indeed, there is only very limited experimental information at lower energies. It consists of measurements of the $\bar{p} p$ annihilation cross section at very low energy and of data on the level shifts and widths of antiproton-proton atoms. 
We have calculated those observables based on our $\bar{N} N$ interaction [20], which can be considered as predictions of our chiral potential. Results for the $\bar{p} p$ annihilation cross section are shown in Fig. 3, where the cross section is multiplied by the velocity $\beta$ of the incoming $\bar{p}$. Values based on the amplitudes of the PWA are also included (filled circles). An interesting aspects of those data is that one can see the anomalous behavior of the reaction cross section near threshold due to the presence of the attractive Coulomb force. Usually the cross sections for exothermic reactions behave like $1 / \beta$ so that $\beta \sigma_{a n n}$ is then practically constant, cf. left side of Fig. 3 for $p_{\text {lab }} \approx 100-300 \mathrm{MeV} / \mathrm{c}$. However, the Coulomb attraction modifies that to a $1 / \beta^{2}$ behavior for energies very close to the threshold.

For illustration we show also predictions for $\bar{n} p$ scattering, see Fig. 4. Data for this reaction were not included in the PWA [18] because they are less precise than the ones for $\bar{p} p$. Nevertheless, the $\bar{n} p$ system is interesting because it is a pure isospin $I=1$ state so that one can test the $I=1$ component of the $\bar{N} N$ amplitude independently.
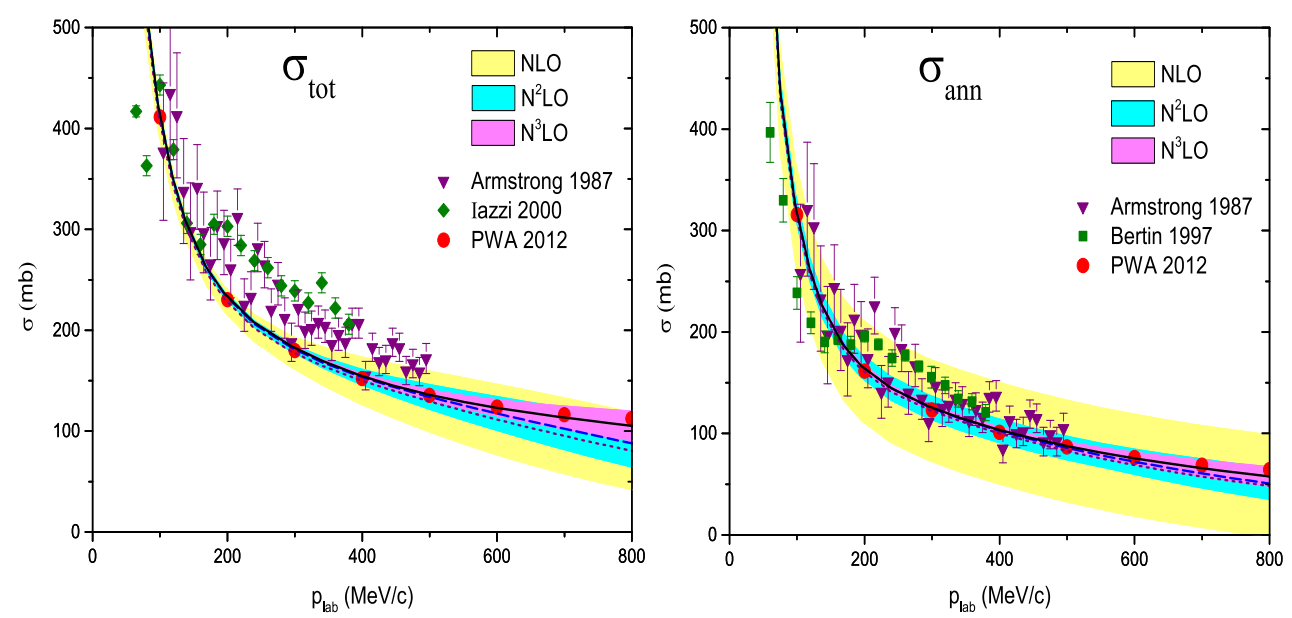

Figure 4. Total $\left(\sigma_{t o t}\right)$ and integrated annihilation $\left(\sigma_{\text {ann }}\right)$ cross sections for $\bar{n} p$ scattering. Same description of curves as in Fig. 2.

Our results for the $\bar{p} p$ atomic level shifts and widths are summarized in Table 1 and compared with measurements [25-28]. In the pertinent calculation the Trueman formula [29] is utilized and it is applied to the theory results with the second-order term taken into account for the $S$-waves. It has been found in Ref. [30] that values obtained in this way agree rather well with direct calculations. In this context let us emphasize that the results in Table 1, including those for the $\mathrm{N}^{2} \mathrm{LO}$ interaction from Ref. [19], are deduced, of course, from a calculation in particle basis. In particular, the Coulomb force in $\bar{p} p$ is taken into account and likewise the $p-n$ mass difference that leads to separated thresholds for the $\bar{p} p$ and $\bar{n} n$ channels. The corresponding results given in our earlier study of the $\bar{N} N$ interaction within chiral EFT [19] are from a calculation in the isospin basis.

Given that experimental evidence on level shifts and widths in antiprotonic hydrogen was not taken into account in the PWA [18], it is remarkable that there is a rather good agreement between our predictions and experiments, especially with the more recently published measurement by Augsburger et al. [26]. In this context, let us mention that additional assumptions have to be made in order to derive the splitting of the ${ }^{1} S_{0}$ and ${ }^{3} S_{1}$ level shifts from the experiment $[28,31]$. This caveat has to be kept in mind when comparing the theory results with experiments. 
Table 1. Hadronic shifts and broadenings in hyperfine states of $\bar{p} \mathrm{H}$ for the chiral potentials with $R=0.9 \mathrm{fm}$. For comparison $\mathrm{N}^{2} \mathrm{LO}$ predictions of our previous chiral potential are included, based on the cutoff combination $(\Lambda, \tilde{\Lambda})=(450,500) \mathrm{MeV}$ [19]. The experimental information is taken from Refs. [25-28].

\begin{tabular}{c||c|c|c|c|c}
\hline \hline & NLO & $\mathrm{N}^{2} \mathrm{LO}$ & $\mathrm{N}^{3} \mathrm{LO}$ & $\mathrm{N}^{2} \mathrm{LO}[19]$ & Experiment \\
\hline \hline$E_{{ } S_{0}}(\mathrm{eV})$ & -448 & -446 & -443 & -436 & $\begin{array}{c}-440(75)[26] \\
-740(150)[25]\end{array}$ \\
$\Gamma_{{ } S_{0}}(\mathrm{eV})$ & 1155 & 1183 & 1171 & 1174 & $1200(250)[26]$ \\
& & & & & $1600(400)[25]$ \\
\hline$E_{3 S_{1}}(\mathrm{eV})$ & -742 & -766 & -770 & -756 & $-785(35)[26]$ \\
& & & & & $-850(42)[27]$ \\
$\Gamma_{{ }^{3} S_{1}}(\mathrm{eV})$ & 1106 & 1136 & 1161 & 1120 & $940(80)[26]$ \\
& & & & & $770(150)[27]$ \\
\hline$E_{3} P_{0}(\mathrm{meV})$ & 17 & 12 & 8 & 16 & $139(28)[28]$ \\
$\Gamma_{3 P_{0}}(\mathrm{meV})$ & 194 & 195 & 188 & 169 & $120(25)[28]$ \\
\hline \hline$E_{1 S}(\mathrm{eV})$ & -670 & -688 & -690 & -676 & $-721(14)[26]$ \\
$\Gamma_{1 S}(\mathrm{eV})$ & 1118 & 1148 & 1164 & 1134 & $1097(42)[26]$ \\
\hline$E_{2 P}(\mathrm{meV})$ & 1.3 & 2.8 & 4.7 & 2.3 & $15(20)[28]$ \\
$\Gamma_{2 P}(\mathrm{meV})$ & 36.2 & 37.4 & 37.9 & 27 & $38.0(2.8)[28]$ \\
\hline \hline
\end{tabular}

Table 2. Bound states and resonances (in MeV) predicted by recently published $\bar{N} N$ potentials. In Ref. [11] only the ${ }^{1} S_{0}$ partial wave is considered.

\begin{tabular}{c||c|c|c|c}
\hline \hline & $\mathrm{N}^{2} \mathrm{LO}[19,34]$ & El-Bennich [14] & Entem [15] & Milstein [11] \\
\hline \hline${ }^{11} S_{0}$ & - & $-4.8-\mathrm{i} 26$ & - & $22-\mathrm{i} 33$ \\
${ }^{31} S_{0}$ & $-37-\mathrm{i} 47$ & - & - & - \\
${ }^{13} S_{1}$ & $+(4.8 \cdots 21.3)-\mathrm{i}(60.6 \cdots 74.9)$ & - & - & \\
${ }^{11} P_{1}$ & - & $1877 \pm \mathrm{i} 13$ & - & \\
${ }^{13} P_{0}$ & $-(3.7 \cdots 0.2)-\mathrm{i}(22.0 \cdots 26.4)$ & $1876 \pm \mathrm{i} 5$ & $1895 \pm \mathrm{i} 17$ & \\
${ }^{33} P_{0}$ & - & $1871 \pm \mathrm{i} 11$ & - & \\
${ }^{13} P_{1}$ & - & $1872 \pm \mathrm{i} 10$ & - & \\
${ }^{33} P_{1}$ & - & $-4.5-\mathrm{i} 9$ & - & \\
\hline \hline
\end{tabular}

One of the topics that has escorted studies of the $\bar{N} N$ interaction over many decades is the possible existence of $\bar{N} N$ bound states, also called baryonium states, see, e.g., Ref. [32] for some historical remarks. So far there is no clear and generally accepted evidence for their existence. Nonetheless, let me summarize some recent predictions for $\bar{N} N$ bound states and resonances in Table 2 for curiosity reasons. Besides our results from the $\mathrm{N}^{2} \mathrm{LO}$ potential in [19], those of a meson-exchange potential [14] and an $\bar{N} N$ interaction based on the constituent quark model [15] are included. 
In the table the spectroscopic notation ${ }^{(2 I+1)(2 S+1)} L_{J}$ is used for identifying the partial waves. In case of bound states the energy $E_{B}$ with respect to the $\bar{p} p$ threshold is given, $M_{p}+M_{\bar{p}}=$ 1876.574 MeV, while for resonances the total energy is provided. Notice that there are cases like that of the ${ }^{13} S_{1}-{ }^{13} D_{1}$ state predicted by the $\mathrm{N}^{2} \mathrm{LO}$ interaction from Ref. [19], or the ${ }^{11} S_{0}$ state given in Ref. [11], where there is a pole which corresponds to a positive value of the binding energy! The positive sign of the real part of $E_{B}$ indicates that the poles are actually located above the $\bar{N} N$ threshold. But they move below the threshold when the imaginary part of the potential is switched off and that is the reason why they are referred to as bound states. To be precise these are unstable bound states in the terminology of Ref. [33]. Since those poles lie on the physical sheet they do not correspond to resonances.

Evidently, and not unexpectedly, conclusions are difficult to draw given the large spread of the results. Indeed, the only "unambiguous" candidate could be the state in the ${ }^{13} P_{0}$ partial wave where all potentials predict either a bound state or a resonance. It should be said that the bound states in the ${ }^{1} S_{0}$ partial waves in Refs. [11,34] are primarily a result of attempting to reproduce the $\bar{p} p$ invariant mass spectrum measured in the reaction $J / \psi \rightarrow \gamma \bar{p} p$ by the BESIII Collaboration [4], which exhibits a strong near-threshold enhancement. However, the obtained results for the binding energy differ significantly, and even appear in different isospin states. Moreover, neither of them seems to correspond to the $X(1835)$ (also denoted as $X(\bar{p} p)$ ), a state that the BESIII Collaboration itself sees as being possibly responsible for the enhancement of the $\bar{p} p$ mass spectrum $[4,35]$. Finally, note that the width of the $S$-wave states, $\Gamma=-2 \operatorname{Im} E_{B}$, are in general rather large.

\section{Summary}

In this contribution I have presented results of a study of the antinucleon-nucleon interaction within chiral effective field theory $[19,20]$. This novel approach suggested by Weinberg has been applied rather successfully to the nucleon-nucleon interaction and can be adapted straightforwardly to the antinucleon-nucleon system. So far the antinucleon-nucleon potential has been derived up to next-tonext-to-next-to-leading order in the chiral expansion. The low-energy constants associated with the arising contact interactions are fixed by a fit to phase shifts and inelasticities provided by a recently published phase-shift analysis of antiproton-proton scattering data. The achieved description of the antinucleon-nucleon amplitudes is excellent and of a quality comparable to the one found in case of the nucleon-nucleon interaction at the same order. As a special application of the antinucleon-nucleon potential predictions for the level shifts and width of antiproton-proton atoms have been presented. Further applications of our chiral $\bar{N} N$ potentials, notably to the reactions $J / \psi \rightarrow \gamma \bar{p} p$ [34], $e^{+} e^{-} \rightarrow \bar{p} p$ [36], and $e^{+} e^{-} \rightarrow 5 \pi, 6 \pi$ [37], can be found in the literature.

\section{Acknowledgements}

I would like to thank Ling-Yun Dai, Xian-Wei Kang, and U.-G. Meißner for collaboration on the topics covered by my talk. This work is supported in part by the DFG and the NSFC through funds provided to the Sino-German CRC 110 "Symmetries and the Emergence of Structure in QCD" and by the BMBF (contract No. 05P2015 -NUSTAR R\&D).

\section{References}

[1] J. Z. Bai et al. [BES Collaboration], Phys. Rev. Lett. 91, 022001 (2003).

[2] B. Aubert et al. [BaBar Collaboration], Phys. Rev. D 72, 051101 (2005). 
[3] B. Aubert et al. [BaBar Collaboration], Phys. Rev. D 73, 012005 (2006).

[4] M. Ablikim et al. [BESIII Collaboration], Phys. Rev. Lett. 108, 112003 (2012).

[5] A. Sibirtsev, J. Haidenbauer, S. Krewald, U.-G. Meißner and A. W. Thomas, Phys. Rev. D 71, 054010 (2005).

[6] B. Loiseau and S. Wycech, Phys. Rev. C 72, 011001 (2005).

[7] J. Haidenbauer, U.-G. Meißner and A. Sibirtsev, Phys. Rev. D 74, 017501 (2006).

[8] D. R. Entem and F. Fernández, Phys. Rev. D 75, 014004 (2007).

[9] J.-P. Dedonder, B. Loiseau, B. El-Bennich, and S. Wycech, Phys. Rev. C 80, 045207 (2009).

[10] J. Haidenbauer and U.-G. Meißner, Phys. Rev. D 86, 077503 (2012).

[11] A. I. Milstein and S. G. Salnikov, Nucl. Phys. A 966, 54 (2017).

[12] W. Erni et al. [Panda Collaboration], arXiv:0903.3905 [hep-ex].

[13] V. Barone et al. [PAX Collaboration], hep-ex/0505054.

[14] B. El-Bennich, M. Lacombe, B. Loiseau and S. Wycech, Phys. Rev. C 79, 054001 (2009).

[15] D.R. Entem and F. Fernández, Phys. Rev. C 73, 045214 (2006).

[16] G. Y. Chen and J. P. Ma, Phys. Rev. D 83, 094029 (2011).

[17] E. Epelbaum, H.-W. Hammer and U.-G. Meißner, Rev. Mod. Phys. 81, 1773 (2009).

[18] D. Zhou and R. G. E. Timmermans, Phys. Rev. C 86, 044003 (2012).

[19] X. W. Kang, J. Haidenbauer and U.-G. Meißner, JHEP 1402, 113 (2014).

[20] L. Y. Dai, J. Haidenbauer and U.-G. Meißner, JHEP 1707, 078 (2017).

[21] S. Weinberg, Nucl. Phys. B 363, 3 (1991).

[22] E. Epelbaum, W. Glöckle, U.-G. Meißner, Nucl. Phys. A 747, 362 (2005).

[23] E. Epelbaum, H. Krebs and U.-G. Meißner, Eur. Phys. J. A 51, 53 (2015).

[24] R. Machleidt and D. R. Entem, Phys. Rept. 503, 1 (2011).

[25] M. Ziegler et al. [ASTERIX Collaboration], Phys. Lett. B 206, 151 (1988).

[26] M. Augsburger et al., Nucl. Phys. A 658, 149 (1999).

[27] K. Heitlinger et al., Z. Phys. A 342, 359 (1992).

[28] D. Gotta et al., Nucl. Phys. A 660, 283 (1999).

[29] T. L. Trueman, Nucl. Phys. 26, 57 (1961).

[30] J. Carbonell, J.-M. Richard and S. Wycech, Z. Phys. A 343, 325 (1992).

[31] D. Gotta, Prog. Part. Nucl. Phys. 52, 133 (2004).

[32] E. Klempt, F. Bradamante, A. Martin and J.-M. Richard, Phys. Rept. 368, 119 (2002).

[33] A. M. Badalian, L. P. Kok, M. I. Polikarpov, Y. A. Simonov, Phys. Rep. 82, 31 (1982).

[34] X. W. Kang, J. Haidenbauer and U.-G. Meißner, Phys. Rev. D 91, 074003 (2015).

[35] M. Ablikim et al. [BESIII Collaboration], Phys. Rev. Lett. 117, 042002 (2016).

[36] J. Haidenbauer, X.-W. Kang and U.-G. Meißner, Nucl. Phys. A 929, 102 (2014).

[37] J. Haidenbauer, C. Hanhart, X. W. Kang and U.-G. Meißner, Phys. Rev. D 92, 054032 (2015). 